\title{
Chemical association and electronic structure: A new theoretical approach
}

Cite as: J. Chem. Phys. 108, 10175 (1998); https://doi.org/10.1063/1.476476

Submitted: 23 December 1997 . Accepted: 18 March 1998 . Published Online: 10 June 1998

E. Lomba, J. L. López-Martín, and M. Holovko

\section{Lock-in Amplifiers up to $600 \mathrm{MHz}$}
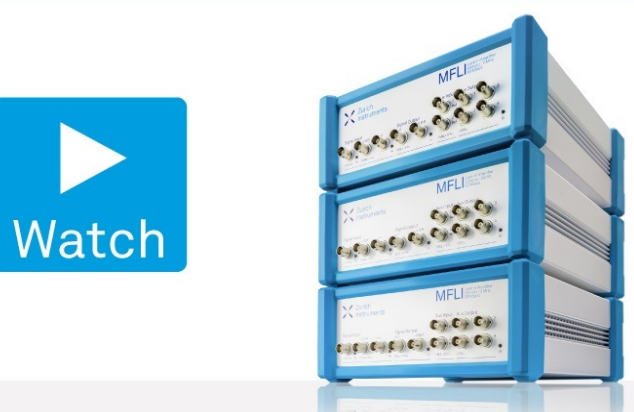


\title{
Chemical association and electronic structure: A new theoretical approach
}

\author{
E. Lomba and J. L. López-Martín \\ Instituto de Química Física Rocasolano, CSIC, Serrano 119, E-28006 Madrid, Spain \\ M. Holovko \\ Institute for Physics of Condensed Matter, Svientsitskoho 1, 290011 Lviv, Ukraine
}

(Received 23 December 1997; accepted 18 March 1998)

\begin{abstract}
Chemical association introduces essential modifications in the microscopic structure of disordered materials, as new species are formed and the density of reactants decreases. These radical changes in the microscopic structure cannot be adequately captured by the pair distribution function, and hence descriptions of the electronic structure at the level of the effective medium approximation break down. We here propose a novel approach closely connected with the single superchain/ effective medium approximation, in which the effects of chemical association are explicitly built-in. The new approximation has been tested in a simple minded model of alkali metal, namely a hard sphere fluid with a one-level tight-binding Hamiltonian with transfer matrix elements modeled by Yukawa terms. This fluid is thought to undergo a dimerization process to yield hard dumbbells via an intermediate stage in which a mixture is present. The proposed approach reproduces the band structure obtained by direct diagonalization for various stages along the reaction path, while retaining the simplicity of a linear theory. (c) 1998 American Institute of Physics. [S0021-9606(98)50624-1]
\end{abstract}

\section{INTRODUCTION}

In a recent paper, two of the authors in collaboration with Anta, Høye, and Kahl, ${ }^{1}$ explored the abilities of the single superchain/effective medium approximation (SSCA/ EMA) to give a correct account of the electronic structure of two covalent elements with well-established tight-binding model Hamiltonians. In Ref. 1 it was found that the SSCA/ EMA-with or without nonlinear correction terms-turned out to yield an accurate description for liquid silicon (a sixfold coordinated material) whereas its performance for liquid carbon, where linear chains and three-fold coordinations are dominant, was rather poor. This failure was associated to the inability of the pair distribution function, which incorporates the microscopic structure into the SSCA/EMA equations, to represent properly the open structures inherent to materials like carbon, let alone elements of group VIa, like Se. The use of the triplet correlation functions to circumvent these limitations poses so far unsurmountable difficulties and hence it is clear that a suitable theoretical alternative is still badly needed.

It is obvious from the above considerations that the underlying problem is the lack of adequate means to deal with chemical association, i.e., clustering, as far as the electronic structure is concerned. Whereas there has been a considerable amount of work invested on approximations that deal with association on the topological and thermodynamic level of description, ${ }^{2,3}$ we are not aware of any attempt to enter the domain of electronic structure calculations with association in mind.

Here we intend to bridge this gap by incorporating the effects of association explicitly into the equations that determine the electronic structure. In order to test the adequacy of our approach we have eliminated from our test model all unneeded features and thus we are back to the simple hard sphere fluid with a one level tight-binding (TB) Hamiltonian, i.e., the familiar hard sphere with embedded coupled quantum Drude oscillators. ${ }^{4}$ Given the complete separation between translational and electronic degrees of freedom, our quenched liquid picture enables a straightforward modeling of the chemical reaction involved in the association process.

We will therefore regard our hard spheres (monomer $A$ ) to be able to dimerize to yield hard dumbbells $\left(A_{2}\right)$ of a given elongation, $L$, following a homogeneous reaction

$$
A+A \rightarrow A_{2} .
$$

An intermediate stage of the reaction process in which the hard dumbbells and hard spheres coexist will also be considered. Obviously the determination on the electronic structure of the system at each stage of the process in only dependent on the instantaneous microscopic structure. Therefore, we will consider the chemical reaction to be slow enough for a representative number of configurations to enter the ensemble averaging that yields the density of states. This is a more than reasonable assumption.

In what follows, we will show how the direct use of the SSCA/EMA with just pair structure information is completely unable to describe the electronic structure when association is present. Then we will define our new set of equations. Association will enter the approximation explicitly, since we will write down a set of SSCA/EMA equations for a mixture of a hard sphere fluid with a one-level TB Hamiltonian plus a hard sphere fluid with a two-level degenerate and nonorthogonal TB basis in the atomic Hamiltonian. We will show that even retaining the linear character of the approximation, this treatment correctly accounts for the extreme modifications that association induces on the band 

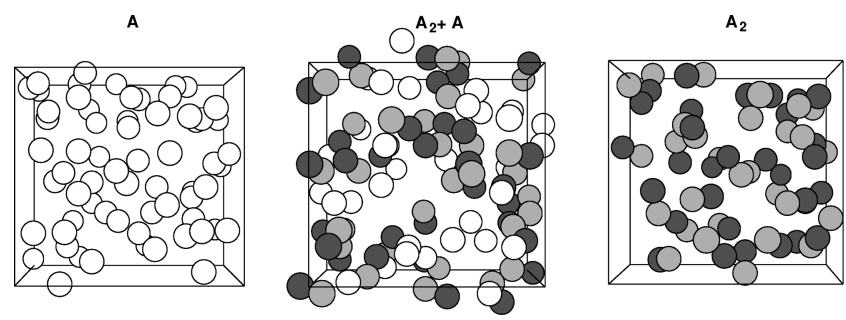

FIG. 1. Snapshots of simulations for a system of hard spheres $(A)$, at $\rho \sigma_{A}=0.375$, hard spheres and hard dumbbells $\left(A_{2}+A\right)$ at $\rho_{A_{2}}=\rho_{A}$ $=0.125$, and hard dumbbells $\left(A_{2}\right)$. When two monomers $A$ (denoted by white spheres) associate to give a dumbbell, light and dark grey colors are assigned to each sphere to denote the newly formed species.

structure. More precisely, if one assigns one electron per atom basis function, like one would have in alkali metals, the dimerization process induces a metal-insulator transition that is correctly described by the theory.

\section{THE HAMILTONIAN MODEL AND THE ATOMIC APPROACH}

As mentioned in the previous section, we will deal with a model in which the translational degrees of freedom are solely controlled by excluded volume effects, the hard sphere repulsion being the only interaction present. The chemical reaction is artificially modeled by considering explicitly the three different stages of the process, namely,

(a) a system of pure hard spheres, with density $\rho_{A} \sigma^{3}$ $=0.375(\sigma$ is the hard sphere diameter and $\rho$ the number density);

(b) an equimolecular mixture of hard spheres and hard dumbbells, the latter particles formed by two fused hard spheres of diameter $\sigma$ separated a distance $L / \sigma$ $=0.6$, with densities $\rho_{A} \sigma^{3}=0.125$ and $\rho_{A_{2}} \sigma^{3}=0.125$;

(c) and finally a system of pure hard dumbbells at density $\rho_{A_{2}} \sigma^{3}=0.1875$. Snapshots of the simulation runs performed for each system can be seen in Fig. 1. Here we have adopted the convention of using light and dark gray to represent the atoms of the $A_{2}$ molecule, and white for isolated atoms.

But the spatial structure is not everything in our system. We also have an electronic structure which we model by means of a one-level tight-binding Hamiltonian in which a single basis function is placed on each atom. In the frame of a TB approach the eigenvalues of the Schrödinger equation satisfy the secular equation

$$
\sum_{i} c_{i}^{(s)}\left(\epsilon_{i}-E^{(s)}\right) \delta_{i j}+\sum_{i \neq j} V_{i j} c_{i}^{(s)}=0,
$$

where $V_{i j}$ is the transfer matrix element between sites $i$ and $j, \epsilon_{i}=V_{i i}$ is the site energy, and the $c_{i}^{(s)}$ coefficients determine the eigenfunction corresponding to the eigenvalue $E^{(s)}$, i.e., $\Psi^{(s)}=\Sigma_{i}{ }^{N} c_{i}^{(s)} \phi_{i}$, where $\left\{\phi_{i}\right\}$ is the atomic basis function set and $\Psi^{(s)}$ is the one-electron eigenfunction, satisfying $H \Psi^{(s)}=E^{(s)} \Psi^{(s)}$. Since we are dealing with a disordered material, the electronic structure will obviously be given by the ensemble average of the eigenvalue distributions.
As to the transfer matrix elements, we have simply chosen Yukawa type interactions that approximately describe the behavior of hopping integrals between $s$ orbitals. Thus

$$
V_{i j}=V\left(r_{i j}\right)=-K \frac{e^{-z r}}{r} .
$$

Here $K$ simply defines the energy scale, and we set $z=1$.

Now, the Green's function theory tells us that the average density of states $(\mathrm{DoS})^{4}$ is given by

$$
D(E)=-\lim _{\varepsilon \rightarrow 0^{+}} \frac{1}{\pi} \operatorname{Im}\{\bar{G}(E+i \varepsilon)\},
$$

where $\bar{G}$ is the average diagonal Green's function, which fulfills the following set of relations,

$$
\begin{aligned}
& z \bar{G}(z)=1+\rho \bar{G}(z)^{2} \int V(r) H(r) d \mathbf{r}, \\
& z=E-\epsilon_{i}+i \varepsilon\left(\varepsilon \rightarrow 0^{+}\right)
\end{aligned}
$$

with $V(r)$ being again the transfer matrix element, and $H(r)$ an electronic analogous of the structural total correlation function, which then also satisfies an Ornstein-Zernike (OZ) relation

$$
H\left(r_{12}\right)=C\left(r_{12}\right)+\rho \bar{G}(z) \int C\left(r_{13}\right) H\left(r_{32}\right) d \mathbf{r}_{3} .
$$

For our "electronic" direct correlation function $C(r)$, Winn and Logan proposed the SSCA/EMA closure

$$
C(r)=g(r) V(r)+[g(r)-1](H(r)-C(r)) .
$$

The system of integral equations can be solved iteratively by the procedures indicated in Ref. 5. The closure (2.6) is where the fluid structure enters the theoretical scheme. At this point we will focus on the fully associated system, i.e., the hard dumbbell. The $g(r)$ is simply the atom-atom distribution function, which is available as a byproduct of the simulation needed to obtain the eigenvalue distribution that serves as a reference for our results, plus an additional $\delta$-function term that accounts for the existence of the rigid bond that attaches every atom in the sample to one single neighbor at a separation $r=L$. Thus we have

$$
g(r)=g_{a t-a t}(r)+\frac{1}{4 \pi r^{2} \rho} \delta(r-L),
$$

where $\delta(r-L)$ is a one-dimensional $\delta$-function. Since this term enters Eq. (2.6) and hence it has to be Fourier transformed to enter the OZ convolution, it produces an oscillating and slowly decaying $\widetilde{C}(k)$, by which some care must be taken in the numerical procedure. Nonetheless, the main results, which are here plotted in Fig. 2, remain essentially unchanged even if one approximates the $\delta$ function by a simple high and narrow peak around $r=L$. What one sees in Fig. 2 is exactly what was found in Ref. 1 for liquid carbon. The SSCA/EMA simply averages out the structure of $D(E)$ and the peaks are lost. The only effect that the $g(r)$ incorporates in $D(E)$ is the change in the band width, which is 


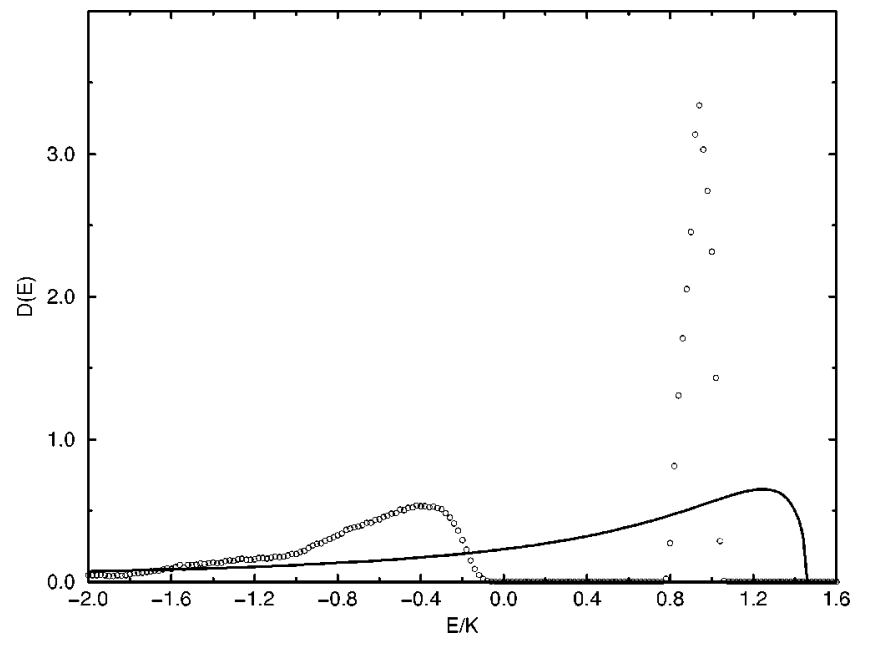

FIG. 2. Electronic density of states for a system of hard dumbbells with a one-level TB Hamiltonian with one basis function per atom and two degenerate basis functions per molecule. Simulation data are denoted by hollow circles and simple (atomic) SSCA/EMA results by a solid line.

larger than that of the nonassociated system as will be seen below, since the presence of neighbors at $r / \sigma=0.6$ implies a larger hopping integral.

Thus, clearly this purely pairwise approach is insufficient, and we still know too little concerning three-body correlation functions to improve the approximations using higher-order terms from Winn and Logan's formalism. ${ }^{4}$

\section{THE ASSOCIATED MODEL: A NEW SET OF EQUATIONS}

On the other hand we can treat explicitly both reactants (monomer $A$ ) and products $\left(\operatorname{dimer} A_{2}\right)$ in the DoS equations,
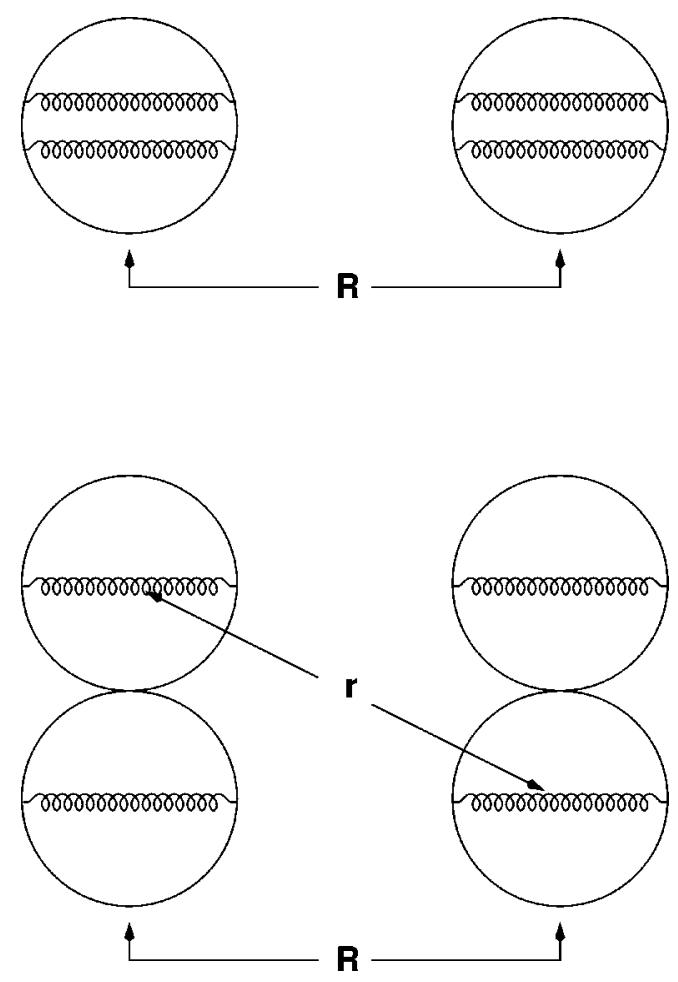

FIG. 3. The hard sphere two-level model versus the hard dumbbells model. See text following Eq. (3.5) for an explanation. if we recall that we are just dealing with a mixture of a one-level species and a two-level system, $A_{2}$. The SSCA/ EMA equations for such a mixture are

$$
\mathbf{H}\left(r_{12}\right)=\mathbf{C}\left(r_{12}\right)+\int \mathbf{H}\left(r_{13}\right)[\rho \overline{\mathbf{G}}(z)] \mathbf{C}\left(r_{32}\right) d \mathbf{r}_{3},
$$

with $[\rho \overline{\mathbf{G}}(z)]_{a b}^{\alpha \beta}=\rho_{a} G_{a b}^{\alpha \beta}(z) \delta_{a b}, \mathbf{X}=\mathbf{V}, \mathbf{C}, \mathbf{H}$,

$$
\mathbf{X}=\left(\begin{array}{ccc}
X_{a a}^{\alpha \alpha} & X_{a a}^{\alpha \beta} & X_{a b}^{\alpha \gamma} \\
X_{a a}^{\beta \alpha} & X_{a a}^{\beta \beta} & X_{a b}^{\beta \gamma} \\
X_{b a}^{\gamma \alpha} & X_{b a}^{\gamma \beta} & X_{b b}^{\gamma \gamma}
\end{array}\right),
$$

and

$$
\overline{\mathbf{G}}(z)=[\mathbf{z}-\mathbf{S}(z)]^{-1},
$$

with

$$
\begin{aligned}
S_{a a}^{\alpha \beta}= & \sum_{\varepsilon=1}^{2} \sum_{\delta=1}^{2} \int V_{a a}^{\alpha \varepsilon}(r) \rho_{a} \bar{G}_{a a}^{\varepsilon \delta}(z) H_{a a}^{\delta \beta}(r) d \mathbf{r} \\
& +\int V(r)_{a b}^{\alpha \gamma} \rho_{b} \bar{G}_{b b}^{\gamma \gamma}(z) H_{b a}^{\gamma \beta}(r) d \mathbf{r}, \\
S_{b b}^{\gamma \gamma}= & \sum_{\varepsilon=1}^{2} \sum_{\delta=1}^{2} \int V_{b a}^{\alpha \varepsilon}(r) \rho_{a} \bar{G}_{a a}^{\varepsilon \delta}(z) H_{a b}^{\delta \beta}(r) d \mathbf{r} \\
& +\int V(r)_{b b}^{\gamma \gamma} \rho_{b} \bar{G}_{b b}^{\gamma \gamma}(z) H_{b b}^{\gamma \gamma}(r) d \mathbf{r},
\end{aligned}
$$

together with the SSCA/EMA closure

$$
\begin{aligned}
C_{a b}^{\alpha \beta}(r)= & g_{a b}^{\alpha \beta}(r) V_{a b}^{\alpha \beta}(r)+\left[g_{a b}^{\alpha \beta}(r)-1\right]\left(H_{a b}^{\alpha \beta}(r)\right. \\
& \left.-C_{a b}^{\alpha \beta}(r)\right) .
\end{aligned}
$$

In these equations the Latin subscripts denote the species and Greek superscripts refer to the atomic basis function involved. Note that when the particle is a dimer, two possible levels - though degenerate - are available, whereas only one is present in monomers. Besides, the submatrix $\mathbf{z}^{A_{2} A_{2}}$ has a rather peculiar structure; normally, one would encounter a diagonal energy matrix with values $[\mathbf{z}]_{\alpha \beta}=\left(E-\epsilon_{\alpha}\right) \delta_{\alpha \beta}$, but now it turns out that we have a coupling term between the two electronic states in the same $\operatorname{dimer} A_{2}$, by which in the two-level model one finds

$$
\left[\mathbf{z}^{A_{2} A_{2}}\right]_{\alpha \beta}=E \delta_{\alpha \beta}-\epsilon_{\alpha \beta} .
$$

Since we only have one type of atomic function, i.e., they are degenerate, $\epsilon=\epsilon_{\alpha \alpha}=\epsilon_{\beta \beta}$ (and we take $\epsilon=0$ ). Now $\epsilon_{\alpha \beta}$ $=-K e^{-z L} / L$, which is precisely the coupling between the two intramolecular electronic degrees of freedom. We have therefore simplified the problem in the way indicated in Fig. 3. The original problem was that shown in the lower figure (the electronic levels are represented by oscillators) and we have performed a reduction to the two-level system shown in the upper figure. It is interesting to compare the eigenvalues that would result from both two-particle systems. For the two-level hard spheres one gets 


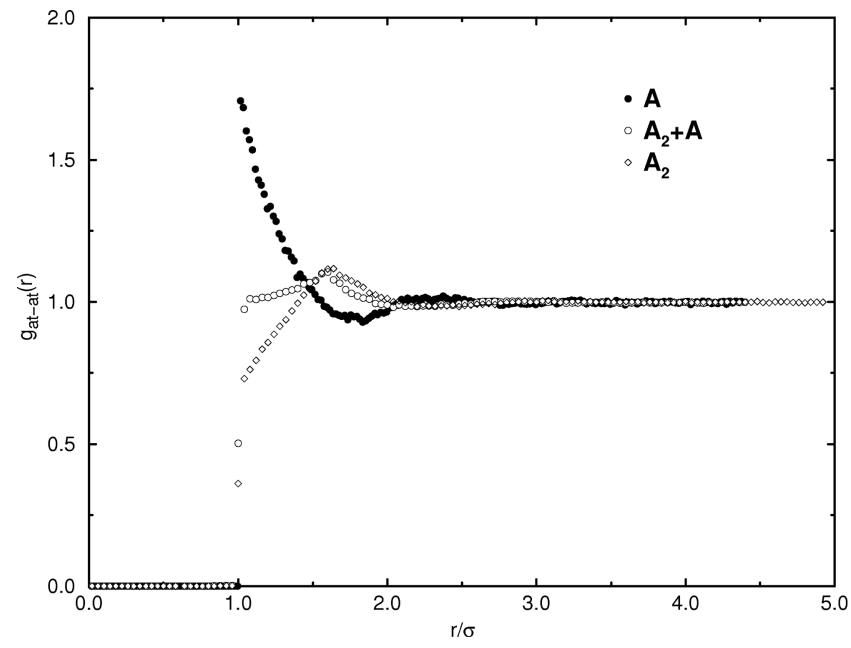

FIG. 4. Atom-atom correlation functions for the hard sphere fluid, the hard sphere and hard dumbbell mixture, and the pure hard dumbbell fluid. Note that for the hard dumbbell correlations we have omitted the $\delta$ function term located at $L=0.6 \sigma_{A}$.

$$
\begin{aligned}
& E_{1}=-V(L), \\
& E_{2}=-V(L), \\
& E_{3}=V(L)-2 V(R), \\
& E_{4}=V(L)+2 V(R),
\end{aligned}
$$

whereas the two dumbbells yield

$$
\begin{aligned}
& E_{1}=-V(L)+V(R)-V(r), \\
& E_{2}=-V(L)-V(R)+V(r), \\
& E_{3}=V(L)-V(R)-V(r), \\
& E_{4}=V(L)+V(R)+V(r) .
\end{aligned}
$$

Thus one immediately appreciates that unless the different cross interaction (due to the difference between $r$ and $R$ ) is taken into account one will get a Dirac $\delta$ line at $E$ $=-V(L)$ in the simplified model, whereas in Fig. 2 it can be seen that the dumbbells generate a high-energy peak that has a finite width. It is also apparent that the spread of the eigenvalues will be larger as the difference between $r$ and $R$ is bigger, and also that the height of this high energy peak, i.e., the density of eigenvalues, will be much larger than the lowenergy band.

Therefore in this work we have accounted for the asymmetry in the cross interaction through a somewhat tedious but efficient procedure, thus compensating the difference between the two models of Fig. 3. First, in Eqs. (3.1)-(3.4) one incorporates the fluid structure though the pair distribution functions which we have just taken from simulation (shown in Fig. 4). But now, additionally the $A_{2}-A_{2}$ term has to reflect the orientational dependence of the problem. To be precise the $A_{2}-A$ term would also need to incorporate this dependence, but the effect is much smaller and therefore here we only will concentrate on the $A_{2}-A_{2}$ contribution. A complete treatment of the orientational dependence would require us to add the angular dependence to all the correlation functions, and introduce the angular averaging and convolu- tions in Eqs. (3.1) and (3.2), incorporating spherical harmonic expansions to handle them. We will, however, see that a more simple-minded approach is more than enough for an accurate description. If one represents a given set of relative orientations between two dumbbells in an intermolecular reference frame $\left[\left(\cos \theta_{1}, \cos \theta_{2}, \cos \phi_{12}\right)\right.$ in the usual notation for linear molecules], and calculates the corresponding interactions between the oscillators in our reduced model of a two-level hard sphere, one has an interaction

$$
V_{a b}^{A_{2} A_{2}}\left(r, \cos \theta_{1}, \cos \theta_{2}, \cos \phi_{12}\right)=-K \frac{e^{-z r_{a b}}}{r_{a b}},
$$

with

$$
\begin{aligned}
r_{11}= & r^{2}+\frac{L^{2}}{2}-L r \cos \theta_{1}+L r \cos \theta_{2} \\
& -\frac{L^{2}}{2}\left[\cos \theta_{1} \cos \theta_{2}+\cos \phi_{12} \sin \theta_{1} \sin \theta_{2}\right], \\
r_{12}= & r^{2}+\frac{L^{2}}{2}-L r \cos \theta_{1}-L r \cos \theta_{2}+\frac{L^{2}}{2}\left[\cos \theta_{1} \cos \theta_{2}\right. \\
& \left.+\cos \phi_{12} \sin \theta_{1} \sin \theta_{2}\right], \\
r_{21}= & r^{2}+\frac{L^{2}}{2}+L r \cos \theta_{1}+L r \cos \theta_{2} \\
& +\frac{L^{2}}{2}\left[\cos \theta_{1} \cos \theta_{2}+\cos \phi_{12} \sin \theta_{1} \sin \theta_{2}\right], \\
r_{22}= & r^{2}+\frac{L^{2}}{2}+L r \cos \theta_{1}-L r \cos \theta_{2} \\
& -\frac{L^{2}}{2}\left[\cos \theta_{1} \cos \theta_{2}+\cos \phi_{12} \sin \theta_{1} \sin \theta_{2}\right] .
\end{aligned}
$$

One can solve the equations (3.1)-(3.4) with this interaction to find out that only the upper energy band of $\bar{G}^{A_{2} A_{2}}\left(z ; \omega_{1}, \omega_{2}\right)$ depends on the orientations. Also, to be consistent the pair distribution function must include the angular dependence, i.e., we have used $g_{A_{2} A_{2}}^{\alpha \beta}$ $=g_{A_{2} A_{2}}\left(r, \theta_{1}, \theta_{2}, \phi_{12}\right)$. Here we resorted to Verlet's modified $^{6}$ integral equation, whose results for a given set of orientations are shown in Fig. 5, together with a comparison of the center-to-center $g_{c c}(r)$ versus simulation. The orientations have been chosen according to a six-point GaussLegendre integration rule over $\cos \theta_{1}$ and $\cos \theta_{2}$, and a sixpoint Gauss-Chebyshev rule over $\cos \phi_{12}$. Some of the results for the "angular dependent'" density of states are shown in Fig. 6.

The extreme cases correspond to nearly parallel (solid line) and nearly head-to-tail (dashed line) alignments. Notice that the head-to-tail alignment leads to a larger spread in the eigenvalues, as one would expect from the two-particle eigenvalues of Eqs. (3.6). The more dissimilar the cross interaction, the larger the spread. Now, the total $\bar{G}^{A_{2} A_{2}}(z)$ is calculated by Gaussian integration 


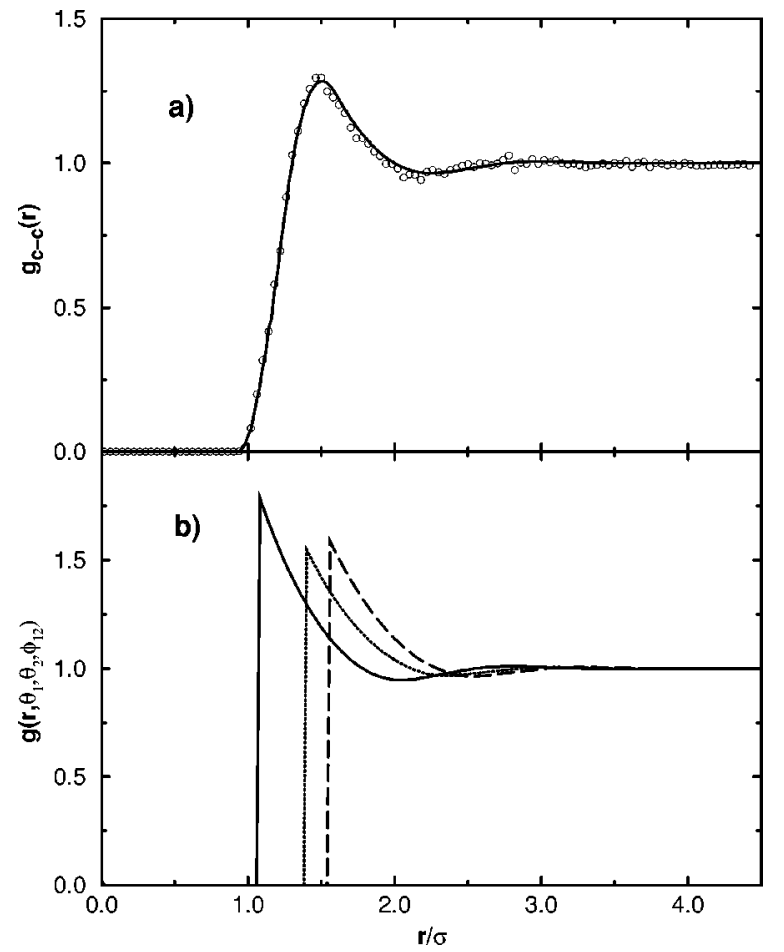

FIG. 5. Distribution functions for the hard dumbbell fluid. The center-tocenter distribution function in the VM approximation (solid line) versus simulation (hollow circles) is shown in the upper figure. In the lower figure the VM result for the molecular pair distribution function for parallel (solid line), head-to-tail (dashed line), and an intermediate configuration (dotted line) are shown.

$$
\begin{aligned}
\bar{G}^{A_{2} A_{2}}(z)= & \frac{8}{\omega^{C}} \sum_{i}^{n_{L}} \sum_{j}^{n_{L}} \sum_{k}^{n_{G}} \frac{1}{\omega_{i}^{G}} \frac{1}{\omega_{j}^{G}} \\
& \times \bar{G}^{A_{2} A_{2}}\left(z ; \cos \theta_{i}, \cos \theta_{j}, \cos \phi_{k}\right),
\end{aligned}
$$

where $\omega^{C}$ and $\omega^{G}$ are the Gauss-Chebyshev and GaussLegendre weights and $\cos \theta_{i}$ and $\cos \phi_{k}$ are the integration nodes defined between 0 and 1 , given the symmetry of the problem.

A summary of the results can be seen in Fig. 7, where theory and simulation are confronted for the pure monomer system $A$, the equimolecular mixture $A_{2}+A$, and the purely diatomic system $A_{2}$. We see that contrary to the atomic approach shown in Fig. 2, the theory now reproduces remarkably well all features of the electronic structure both for the mixture and the hard dumbbell system. If one assigns one electron to each atomic orbital, the results indicate that a transition from a conducting atomic fluid to an insulating diatomic system is taking place along with the chemical reaction. This might well be assimilated with a related transition in alkali metals at high temperatures, when approaching the gas phase. ${ }^{7,8} \mathrm{We}$ see that the antibonding conduction band in the dimer is far above the bonding valence band. It is remarkable that such a simple model can grasp the nature of such crucial transitions, and even more remarkable that a simple restructuring of the SSCA/EMA equations to give account of the association can transform the poor results of Fig. 2 into those of Fig. 7. Nonlinearity has been somehow

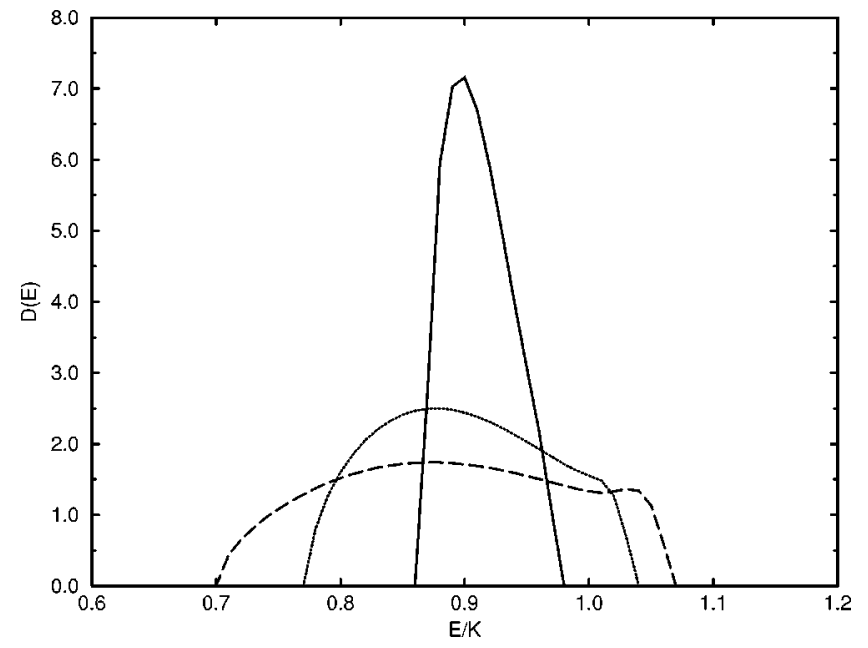

FIG. 6. Contribution to the upper energy antibonding band of the electronic DoS from the hard dumbbell configurations whose pair distribution functions are shown in Fig. 5(b).

built into the equations and thus the closure can retain all its simplicity.

In summary we have presented a simple modification of the SSCA/EMA equation that incorporates explicitly the effect of association. The results clearly indicate that this is a natural way to deal with otherwise complicated band structures. However, if one intends to deal with more complex

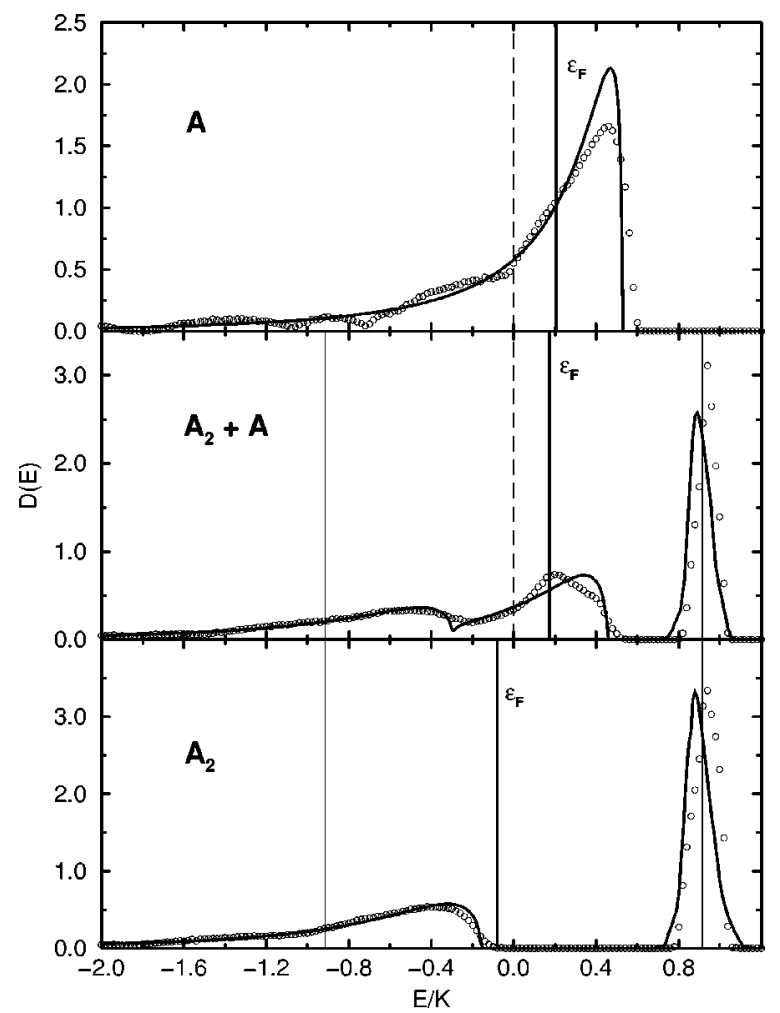

FIG. 7. Electronic density of states for the three stages of the association process (pure monomers, monomers + dimers, pure dimers). Simulation data are indicated by hollow circles and solid lines denote the SSCA/EMA results with built-in association. The site energies are indicated with dashed vertical lines and the resonance energies derived from the intramolecular coupling with thin solid lines. The Fermi energies for half-filled bands are also shown on the figure as solid vertical lines. 
molecules or aggregates, this idea has to be exploited without resorting to the calculation of the molecular distribution function, a task rather involved on itself, and very specially the tedious angular integration. In this respect it might be possible to cast the SSCA/EMA equation into the language of interatomic and intraatomic Green's functions and correlations, to elaborate a (Reference Interaction Site Model) RISM-like EMA equation. Work on this is planned.

\section{ACKNOWLEDGMENTS}

This work has been supported by the Spanish Dirección General de Enseñanza Superior under Grant No. PB94-0112. E.L. is grateful the Ukrainian Academy of Sciences who supported his stay in Lviv during the period while this work was conceived. He also would like to thank the Institute for Phys- ics of Condensed Matter at Lviv for its kind hospitality. M.H. acknowledges support from International Soros Science Education Program trough Grant No. SPU072014.

${ }^{1}$ E. Lomba, J. L. López-Martín, J. A. Anta, J. S. Høye, and G. Kahl, J. Chem. Phys. 106, 10238 (1997).

${ }^{2}$ M. S. Wertheim, J. Stat. Phys. 42, 459 (1986).

${ }^{3}$ Y. V. Kalyuzhnyi, G. Stell, and M. F. Holovko, Chem. Phys. Lett. 235, 355 (1995).

${ }^{4}$ D. E. Logan and M. D. Winn, J. Phys. C 21, 5773 (1988).

${ }^{5}$ E. Lomba and J. L. López-Martín, J. Chem. Phys. 104, 5244 (1996).

${ }^{6}$ S. Labik, W. R. Smith, R. Pospisil, and A. Malijevsky, Mol. Phys. 69, 649 (1990).

${ }^{7} \mathrm{~A}$ description of how dimerization processes are involved in the metal-non metal transition in expanded alkali metals can be found in F. Hensel, J. Phys.: Condens. Matter 2, 33 (1990).

${ }^{8}$ See also F. Hensel and P. Edwards, Phys. World July, 43 (1996). 